\title{
Uptake and impact of vaccinating school age children against influenza during a season with circulation of drifted influenza A and B strains, England, 2014/15
}

RG Pebody ${ }^{1}$, HK Green ${ }^{1}$, N Andrews ${ }^{1}$, NL Boddington ${ }^{1}$, H Zhao ${ }^{1}$, I Yonova ${ }^{23}$, J Ellis ${ }^{1}$, S Steinberger ${ }^{1}$, M Donati ${ }^{1}$, AJ Elliot ${ }^{1}$, HE Hughes $^{1}$, S Pathirannehelage ${ }^{23}$, D Mullett ${ }^{23}$, GE Smith ${ }^{1}$, S de Lusignan ${ }^{23}$, M Zambon ${ }^{1}$

1. Public Health England (PHE), United Kingdom

2. Royal College of General Practitioners Research and Surveillance Unit, Birmingham, United Kingdom

3. University of Surrey, Guildford, United Kingdom

Correspondence: Richard G Pebody (richard.pebody@phe.gov.uk)

Pebody RG, Green HK, Andrews N, Boddington NL, Zhao H, Yonova I, Ellis J, Steinberger S, Donati M, Elliot AJ, Hughes HE, Pathirannehelage S, Mullett D, Smith GE, de Lusignan S, Zambon M. Uptake and impact of vaccinating school age children against influenza during a season with circulation of drifted influenza A and B strains, England, 2014/15. Euro Surveill. 2015;20(39):pii=30029. DOI: http://dx.doi.org/10.2807/1560-7917.ES.2015.20.39.30029

The 2014/15 influenza season was the second season of roll-out of a live attenuated influenza vaccine (LAIV) programme for healthy children in England. During this season, besides offering LAIV to all two to four year olds, several areas piloted vaccination of primary (4-11 years) and secondary (11-13 years) age children. Influenza $\mathrm{A}\left(\mathrm{H}_{3} \mathrm{~N}_{2}\right)$ circulated, with strains genetically and antigenically distinct from the 2014/15 $\mathrm{A}\left(\mathrm{H}_{3} \mathrm{~N}_{2}\right)$ vaccine strain, followed by a drifted $B$ strain. We assessed the overall and indirect impact of vaccinating school age children, comparing cumulative disease incidence in targeted and non-targeted age groups in vaccine pilot to non-pilot areas. Uptake levels were $56.8 \%$ and $49.8 \%$ in primary and secondary school pilot areas respectively. In primary school age pilot areas, cumulative primary care influenza-like consultation, emergency department respiratory attendance, respiratory swab positivity, hospitalisation and excess respiratory mortality were consistently lower in targeted and non-targeted age groups, though less for adults and more severe end-points, compared with non-pilot areas. There was no significant reduction for excess all-cause mortality. Little impact was seen in secondary school age pilot only areas compared with non-pilot areas. Vaccination of healthy primary school age children resulted in population-level impact despite circulation of drifted A and B influenza strains.

\section{Background}

The United Kingdom (UK) started the phased introduction of a universal childhood influenza vaccination programme in the 2013/14 influenza season following the recommendation of the Joint Committee on Vaccination and Immunisation (JCVI) that all healthy children aged two to less than 17 years should be offered the newly licensed live attenuated influenza vaccine (LAIV) [1]. The decision was informed by transmission modelling using Bayesian evidence synthesis, which predicted that vaccination of healthy children would provide direct protection to the vaccinated children themselves and by reducing infection in this group, it would decrease transmission of influenza in the general population and thus provide indirect protection to groups at higher risk of severe disease such as the elderly and those with underlying clinical risk factors [2]. Although North America has a long-standing childhood influenza vaccination programme, there is only limited published observational evidence of whether such programmes produce such indirect population effects [3-5]. Questions also remain as to which paediatric age-groups to target to achieve optimal direct and indirect protection; is it preferable to either vaccinate all school age children or to focus on certain groups such as primary school age children alone?

In the first year of the LAIV programme in England, all healthy children aged two to three years were offered a single dose of LAIV, together with children of primary school age (4-11 years) in a series of geographically discrete pilot areas. Early results suggested that vaccinating primary school age children led to populationlevel reductions for a range of influenza indicators in pilot areas compared with non-pilot areas [6]. These results, however, were not significant, likely due to the low intensity of virus circulation in the 2013/14 influenza season and the relatively limited number of primary school age children vaccinated.

In $2014 / 15$, the national LAIV programme was extended to all two to four year-olds in England [7]. In addition, the primary school age pilots continued with an increase in the size of the target populations where healthy children 4 to 11 years of age were offered a dose of LAIV, together with the recruitment of additional 


\section{FIGURE 1}

Geographical distribution of school-age pilot areas, England, week 402014 to week 142015

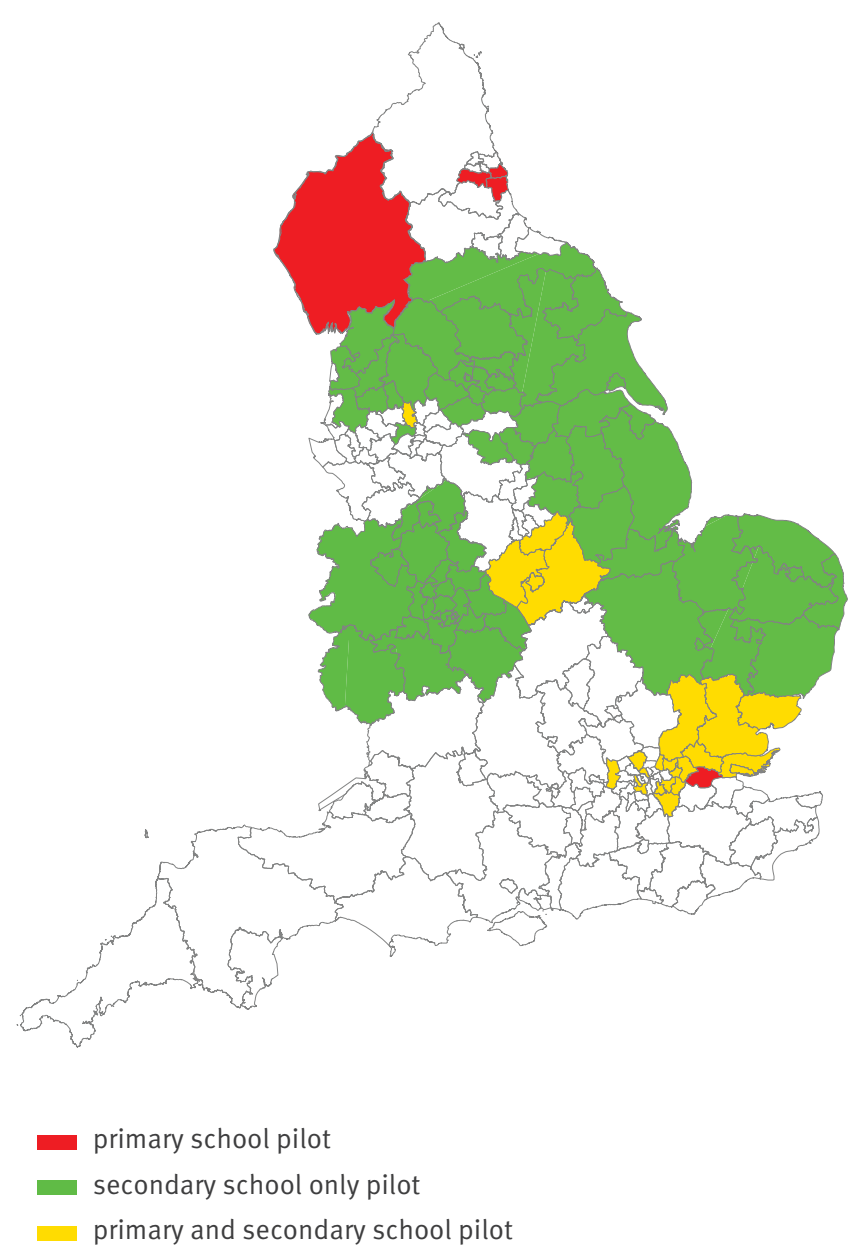

Contains Ordnance Survey data, Crown copyright and database right 2014.

pilot areas where healthy secondary school children aged 11 to 13 years were offered LAIV. A range of delivery models were deployed specifically school-based or within the community via pharmacies and primary care. The 2014/15 influenza season was a moderately intense season dominated initially by the circulation of influenza $A\left(\mathrm{H}_{3} \mathrm{~N}_{2}\right)$ virus, which usually results in severe disease in the elderly, followed by influenza B virus [8]. Virological surveillance found that, as seen elsewhere, the dominant circulating influenza $A\left(\mathrm{H}_{3} \mathrm{~N}_{2}\right)$ and $B$ strains were antigenically and genetically drifted against the relevant components of the 2014/15 seasonal influenza vaccine for the northern hemisphere [9].

The implementation of the primary and secondary school age pilots provided a unique opportunity to assess the level of population protection that vaccinating school age children with LAIV might provide over and above the vaccination of pre-school age children in a season when drifted strains circulated. The aim of this paper is thus to measure the uptake of the programme and evaluate the total and indirect impact of vaccinating healthy children of primary or secondary school age in England in 2014/15.

\section{Methods}

Most areas that undertook vaccination of primary school age children in the $2013 / 14$ season (6/7) decided to continue this activity in $2014 / 15$ [6]. Local National Health Service (NHS) England teams with an interest in running secondary school age pilot influenza immunisation programmes were selected by the national team. Different models of delivery, in particular, school-based and community-based through pharmacy and primary care, were undertaken in these pilots. Most were school-based, with the exception of two area teams following a pharmacy-based model and one local team following a community GP delivery model.

\section{Measuring vaccine uptake}

The target population for delivery was defined as children of primary school age (born between 2 Sep 2003 and 1 Sep 2010; 4 to 11 years old) resident in six pilot areas in England: Cumbria, Greater Manchester, Leicestershire and Lincolnshire, London and Essex, Northumberland, Tyne and Wear. The target population for children of secondary school age (born between 2 Sep 2001 and 1 Sep 2003; 11-13 years of age) were children resident in 12 selected pilot areas (Arden, Birmingham and Black Country, Greater Manchester, East Anglia, Essex, Herefordshire and Worcestershire, Lancashire, London, North Yorkshire and Humber, Shropshire and Staffordshire, South Yorkshire and Bassetlaw, West Yorkshire). Four of the latter sites also ran primary school age programmes. The geographical distribution of these sites is shown in Figure 1.

Local NHS teams responsible for the delivery of the LAIV programme in pilot areas gathered and reported data on vaccine administration to Public Health England (PHE) using a standard proforma through a web-based portal. End-of-season programme uptake was calculated based on the number of children in the target population who were reported to have received at least one dose of influenza vaccine during the campaign period (September 2014 until January 2015). Healthy children and at-risk children in whom the vaccine was not contraindicated were offered LAIV. Inactivated influenza vaccine was offered to at-risk children in whom LAIV was contraindicated.

\section{Measuring school age vaccine programme impact}

The study period for the programme impact calculations was from week 402014 until week 14 2015, the end of notable influenza transmission in the community in the $2014 / 15$ season [8].

LAIV programme impact was defined as the difference in cumulative disease incidence in school age pilots compared with non-pilot areas for the study period. 
Uptake of primary and secondary school age influenza vaccination programme in pilot areas by type of delivery, England, week 402014 to week 142015

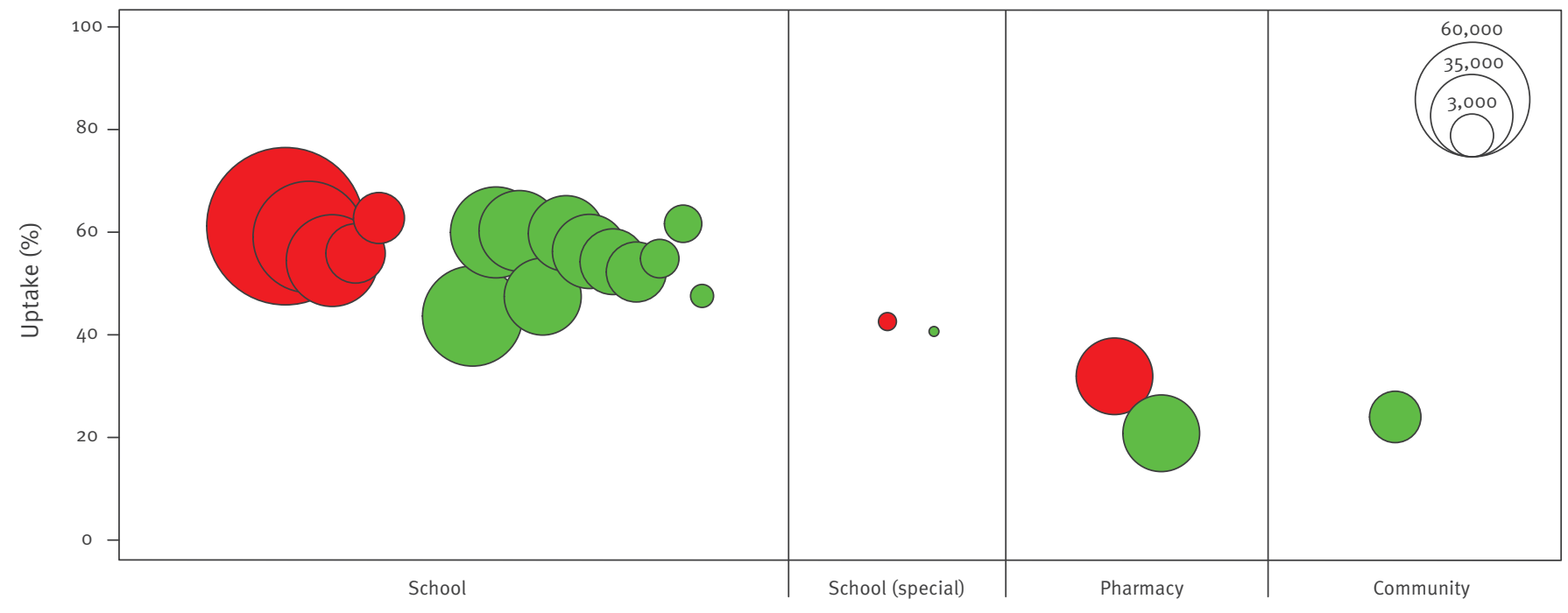

Delivery method

primary school pilot secondary school only pilot

It was measured for a range of clinical and virological respiratory endpoints in primary and secondary care.

Primary school age only vaccine areas were pooled with primary and secondary school age vaccine areas to examine the impact of vaccinating primary school age children together with cohorts of two years of secondary school children in addition to the vaccination of pre-school age children. Secondary school age only pilot areas were compared with non-pilot areas to determine the impact of vaccinating the first two years of secondary school age children alone (i.e. in addition to vaccinating children two to four years of age).

Cumulative levels of activity in pilot versus non-pilot areas were compared for four age groups. To examine direct impact, the two targeted age groups for which surveillance data were available were primary school children (5-10 years old) and secondary school children (11-16 years old, where children aged $11-13$ years were offered vaccine). To examine indirect impact, the non-targeted age groups compared were under 5 years old and 17 years old and older. Overall impact was assessed by comparing the disease incidence for all ages in pilot vaccination areas compared with non-pilot areas. Indirect impact was measured by comparing incidence in non-targeted age groups in pilot relative to non-pilot areas. To ensure appropriate geographical coverage for the sentinel surveillance schemes, additional sites (general practitioners (GPs), emergency departments and hospitals) were recruited in primary and secondary pilot areas where required.

\section{Data sources}

A range of surveillance systems were used to measure the impact of the school age vaccination programme.

\section{Primary care}

Surveillance in primary care was undertaken through monitoring the weekly influenza-like-illness (ILI) consultation rates through the Royal College of General Practitioners (RCGP) Research and Surveillance Centre (RSC) Weekly Returns Service sentinel GP network, with 29 general practices participating in pilot areas and 58 in non-pilot areas. A proportion of these practices, in conjunction with practices recruited through the PHE coordinated Sentinel Microbiology Network $(\mathrm{SMN})$ scheme, undertook respiratory swabbing on patients under 18 years of age presenting with ILI, and a proportion of patients 18 years of age and older.

\section{Secondary care}

The UK Severe Influenza Sentinel Surveillance System (USISS sentinel) consists of a network of 30 NHS hospital trusts ( 15 in pilot areas and 15 in non-pilot areas in 2014/15) who report the weekly number of laboratory-confirmed influenza hospital admissions [10]. Confirmed influenza hospitalisation rates by age group and pilot area were calculated using estimated hospital catchment populations [11]. As age grouping of populations was not consistent with this analysis, agespecific denominator data were estimated using population age-distributions by Strategic Health Authority from the Office for National Statistics [12]. 


\section{FIGURE 3}

Cumulative primary care indicators in primary school pilot, secondary school pilot and non-pilot areas, England, week 40 2014 to week 142015

RCGP ILI

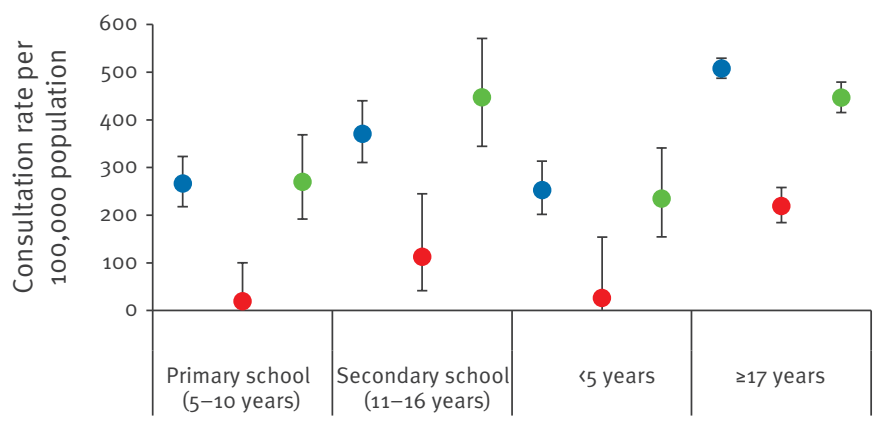

Age group
Sentinel positivity

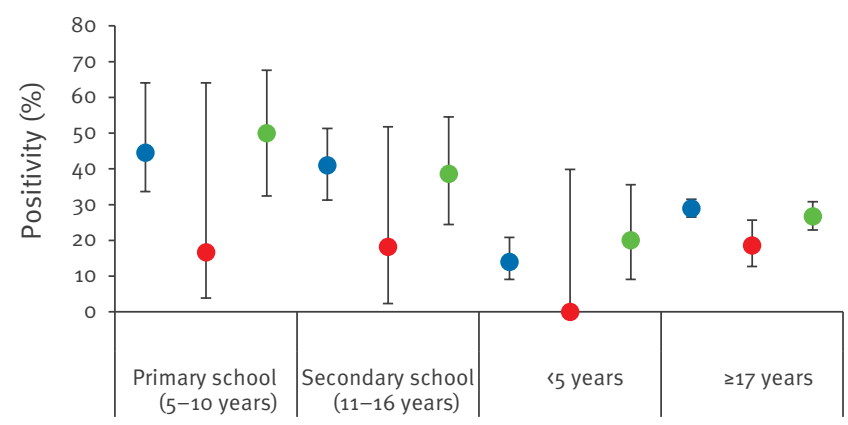

Age group

ILI: influenza-like illness; RCGP: Royal College of General Practitioners.

\section{FIGURE 4}

Cumulative secondary care indicators in primary school pilot, secondary school pilot and non-pilot areas, England, week 40 2014 to week 142015

USISS sentinel

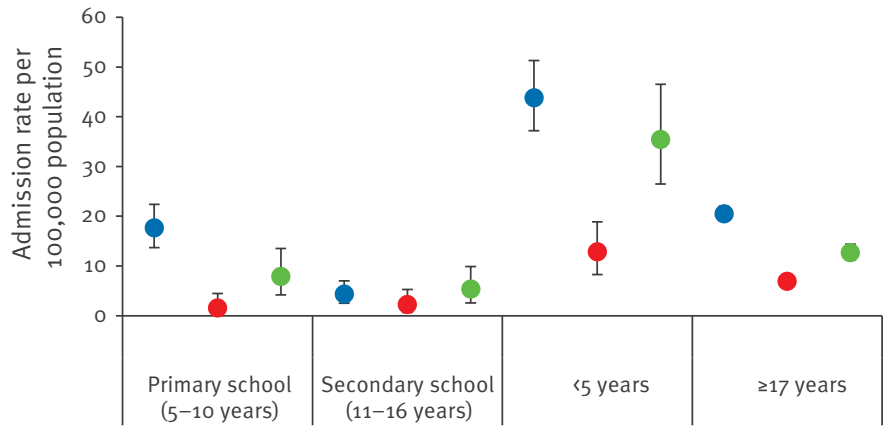

Age group

Datamart

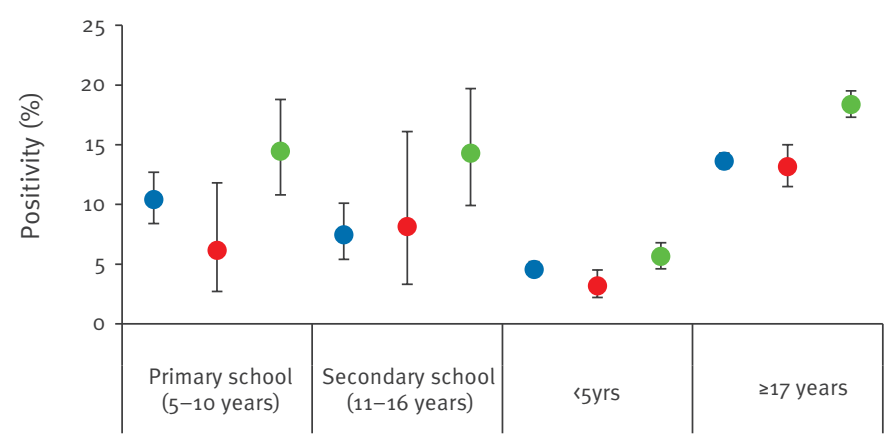

Age group
USISS mandatory

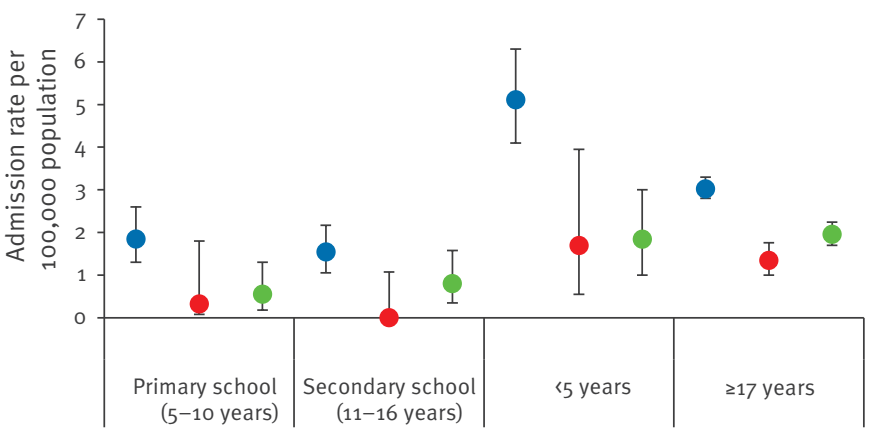

Age group

EDSSS

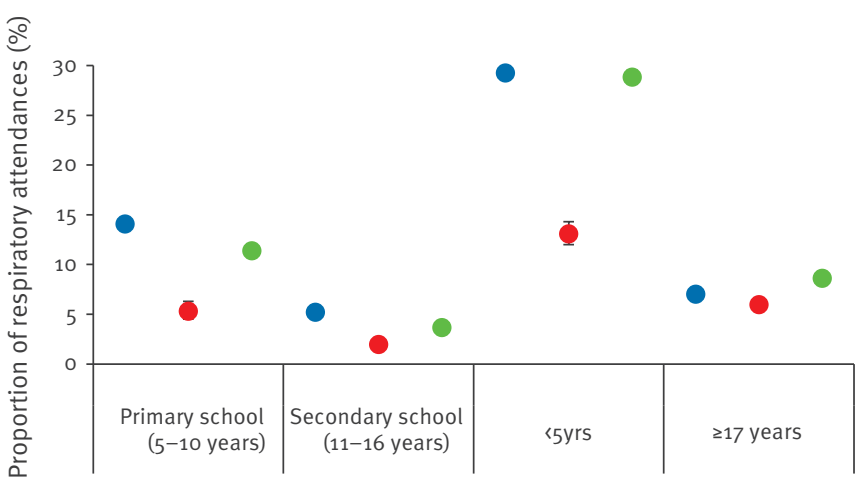

Age group

non-pilot primary school pilot $\quad$ secondary school only pilot $\longmapsto$ exact $95 \%$ confidence interval

EDSSS: Emergency Department Syndromic Surveillance System; USISS: Severe Influenza Sentinel Surveillance System. 


\section{FIGURE 5}

Cumulative weekly all-cause and respiratory excess mortality in primary school pilot, secondary school only pilot and non-pilot areas, England, influenza season, week 402014 to week 142015
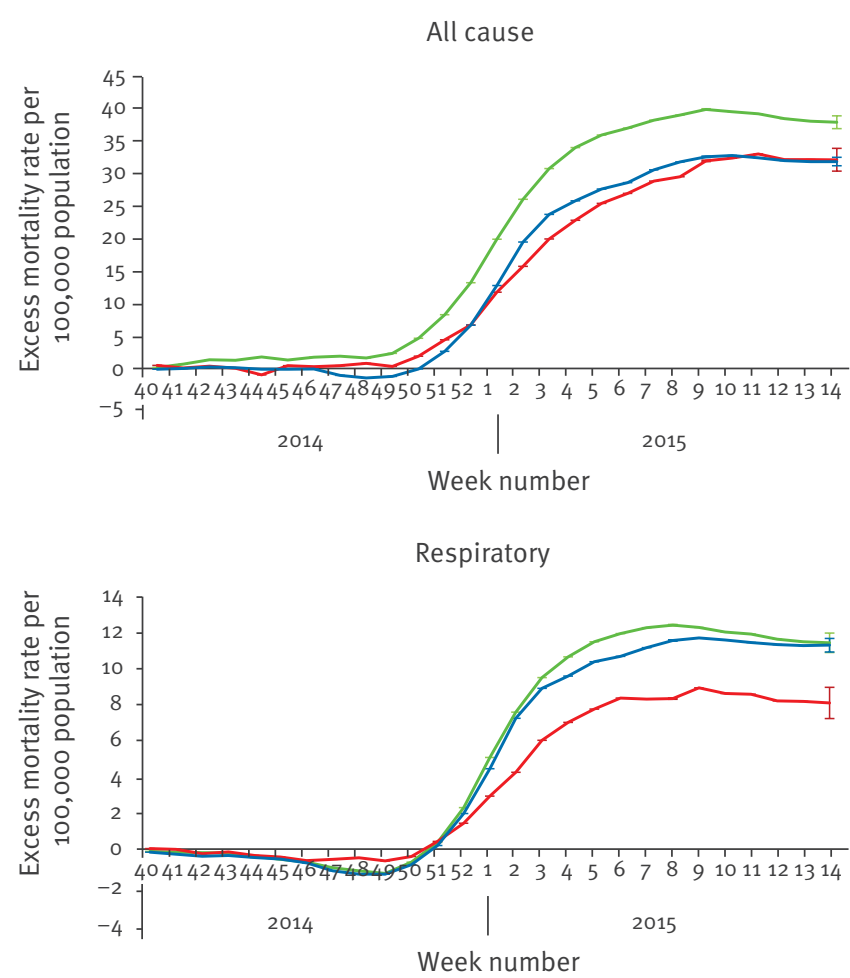

secondary school only pilot $\quad$ non-pilot
primary school

A mandatory scheme is also in operation in all NHS hospitals across England (UK Severe Influenza Surveillance System (USISS mandatory)), monitoring all influenza confirmed intensive care unit (ICU) / high dependency unit (HDU) admissions. Rates were calculated by pilot type and age group as for USISS sentinel [11].

The Respiratory DataMart scheme (RDMS) reports all influenza reverse-transcription-polymerase chain reaction (RT-PCR) respiratory swab results (both positive and negative) from a network of PHE and NHS laboratories in England, with the majority of samples (>90\%) taken from patients in secondary care [13]. Postcode of residence was used to allocate patients to pilot and non-pilot areas. Influenza swab positivity rates in pilot and non-pilot areas were compared by age group.

The Emergency Department Syndromic Surveillance System (EDSSS) monitors routine syndromic surveillance data, in real-time, using anonymised emergency department attendances, across a sentinel network of emergency departments [14]. Attendances monitored include those for respiratory illness. The proportion of all EDSSS attendances for respiratory illness in pilot and non-pilot areas (three emergency departments in

\section{FIGURE 6}

Cumulative, all-age influenza indicators in pilot and nonpilot areas before (2010/11 and 2012/13) and after (2012/13 and 2014/15) vaccine programme introduction, England
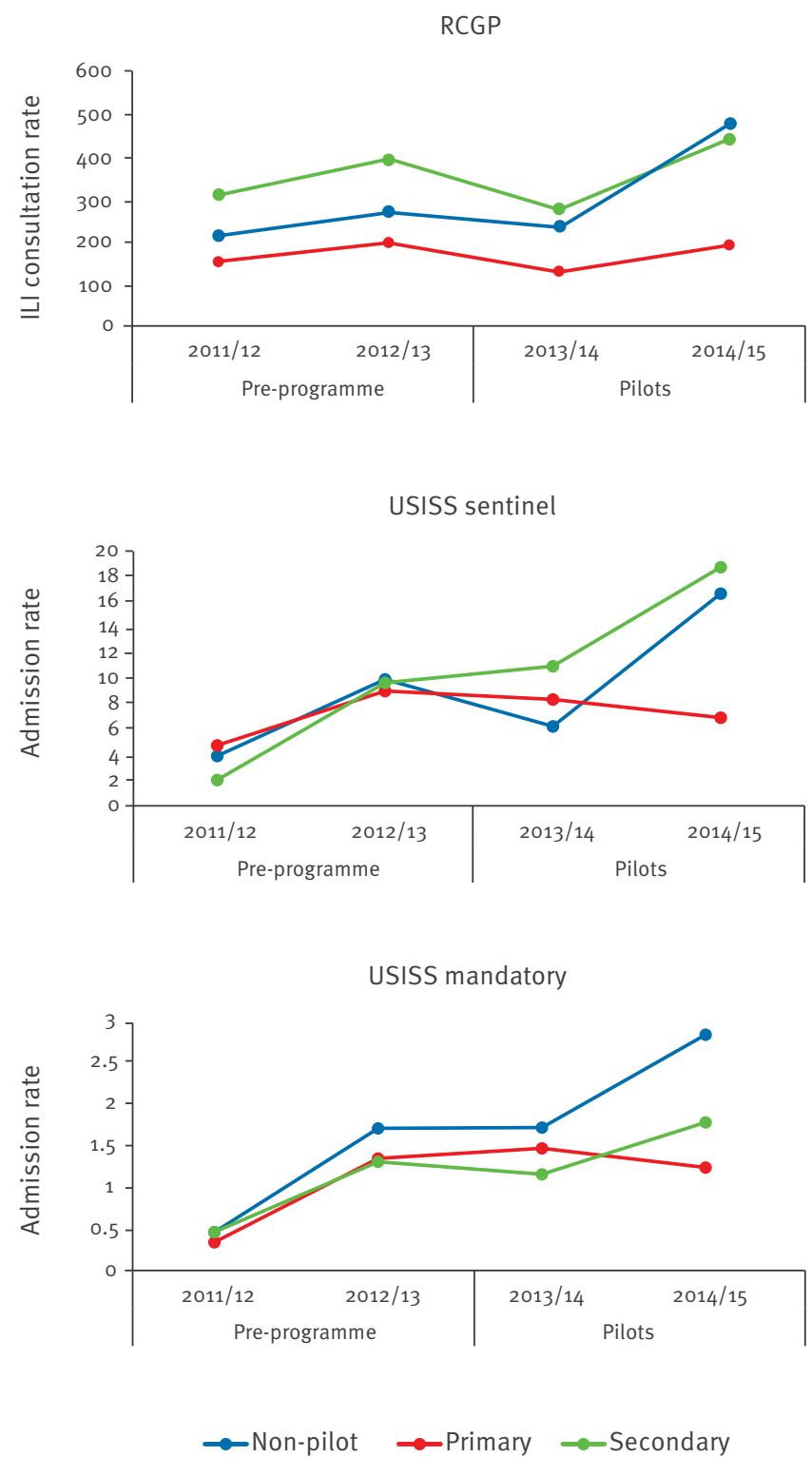

ILI: influenza-like illness; RCGP: Royal College of General Practitioners; USISS: Severe Influenza Sentinel Surveillance System.

Only sites with data available across all four seasons are included.

primary school age pilot areas, eight in secondary and 18 in non-pilot areas) was compared by age group.

Excess mortality

Weekly excess mortality was estimated in pilot and non-pilot areas based on place of residence using routine death registration data from the Office for National Statistics. The European Monitoring of Excess Mortality for Public Health Action (EuroMOMO) standard algorithm was used to calculate the number of deaths expected for a given week in the year [15]. The number 
Impact of vaccinating primary and/or secondary school age children on selected primary care influenza surveillance indicators, England, influenza season, week 402014 to week 142015

\begin{tabular}{|c|c|c|c|c|c|c|c|c|}
\hline \multirow{2}{*}{ Age group } & & \multirow{2}{*}{ Measure } & \multicolumn{3}{|c|}{ RCGP (per 100,000 population) } & \multicolumn{3}{|c|}{ Sentinel swab positivity (\%) } \\
\hline & & & Non-pilot & Primary $^{\mathrm{a}}$ & Secondary & Non-pilot & Primary $^{\mathrm{a}}$ & Secondary \\
\hline \multirow{5}{*}{$\begin{array}{l}\text { Primary } \\
\text { school }\end{array}$} & \multirow{5}{*}{$\begin{array}{l}5^{-10} \\
\text { years }\end{array}$} & Rate/proportion & 266.9 & 19.7 & 269.7 & 44.6 & 16.7 & 50 \\
\hline & & $(\mathrm{n} / \mathrm{N})$ & $(104 / 38,969)$ & $(1 / 5,086)$ & $(39 / 14,459)$ & $37 / 83$ & $1 / 6$ & $17 / 34$ \\
\hline & & Risk difference & & -247 & 3 & & -28 & 5 \\
\hline & & $\begin{array}{c}\text { OR } \\
(95 \% \mathrm{Cl}) \\
\end{array}$ & & $\begin{array}{c}0.06 \\
\text { (0.01 to } 0.62)\end{array}$ & $\begin{array}{c}0.81 \\
\text { (0.39 to 1.69) }\end{array}$ & & $\begin{array}{c}0.25 \\
\text { (0.03 to } 2.22) \\
\end{array}$ & $\begin{array}{c}1.24 \\
\text { (0.56 to } 2.77) \\
\end{array}$ \\
\hline & & $\mathrm{p}$ value & & 0.018 & 0.573 & & 0.213 & 0.594 \\
\hline \multirow{5}{*}{$\begin{array}{l}\text { Secondary } \\
\text { school }\end{array}$} & \multirow{5}{*}{$\begin{array}{l}11-16 \\
\text { years }\end{array}$} & Rate/proportion & 371.22 & 112.6 & 447.1 & 41 & 18.2 & 38.6 \\
\hline & & $(\mathrm{n} / \mathrm{N})$ & $(133 / 35,830)$ & $(6 / 5,330)$ & $(64 / 14,315)$ & $41 / 100$ & $2 / 11$ & $17 / 44$ \\
\hline & & Risk difference & & -259 & 76 & & -23 & -2 \\
\hline & & $\begin{array}{c}\text { OR } \\
(95 \% \mathrm{Cl}) \\
\end{array}$ & & $\begin{array}{c}0.31 \\
\text { (0.10 to } 0.95) \\
\end{array}$ & $\begin{array}{c}0.96 \\
\text { (0.53 to 1.73) }\end{array}$ & & $\begin{array}{c}0.32 \\
\text { (0.07 to 1.56) }\end{array}$ & $\begin{array}{c}0.91 \\
\text { (0.44 to 1.87) }\end{array}$ \\
\hline & & $\mathrm{p}$ value & & 0.04 & 0.882 & & 0.158 & 0.79 \\
\hline \multirow{10}{*}{$\begin{array}{l}\text { Other age } \\
\text { groups }\end{array}$} & \multirow{5}{*}{$<5$ years } & Rate/proportion & 253.1 & 26.1 & 34.6 & 14 & 0 & 20 \\
\hline & & $(\mathrm{n} / \mathrm{N})$ & $(84 / 33,192)$ & $(1 / 3,826)$ & $(27 / 11,511)$ & $21 / 150$ & $0 / 7$ & $8 / 40$ \\
\hline & & Risk difference & & -227 & -219 & & -14 & 6 \\
\hline & & $\begin{array}{c}\text { OR } \\
(95 \% \mathrm{Cl}) \\
\end{array}$ & & $\begin{array}{c}0.08 \\
\text { (0.01 to 1.02) }\end{array}$ & $\begin{array}{c}0.65 \\
(0.25 \text { to } 1.67) \\
\end{array}$ & & 1 & $\begin{array}{c}1.54 \\
\text { (0.62 to } 3.78) \\
\end{array}$ \\
\hline & & $\mathrm{p}$ value & & 0.052 & 0.367 & & NA & 0.351 \\
\hline & \multirow{5}{*}{$\geq 17$ years } & Rate/proportion & 508.1 & 219.1 & 446.6 & 29 & 18.5 & 26.7 \\
\hline & & $(\mathrm{n} / \mathrm{N})$ & $(2,299 / 452,461)$ & $(143 / 65,260)$ & $(767 / 171,735)$ & $378 / 1,305$ & $28 / 151$ & $135 / 506$ \\
\hline & & Risk difference & & -289 & -62 & & -10 & -2 \\
\hline & & $\begin{array}{c}\text { OR } \\
(95 \% \mathrm{Cl}) \\
\end{array}$ & & $\begin{array}{c}0.41 \\
\text { (0.19 to } 0.86) \\
\end{array}$ & $\begin{array}{c}0.67 \\
\text { (0.42 to 1.07) }\end{array}$ & & $\begin{array}{c}0.68 \\
\text { (0.37 to 1.24) } \\
\end{array}$ & $\begin{array}{c}1.23 \\
\text { (0.72 to 2.11) } \\
\end{array}$ \\
\hline & & $\mathrm{p}$ value & & 0.018 & 0.092 & & 0.206 & 0.455 \\
\hline
\end{tabular}

$\mathrm{Cl}$ : confidence interval; NA: not available; OR: odds ratio; RCGP: Royal College of General Practitioners.

a Includes primary and secondary school pilot areas.

of observed deaths (corrected for reporting delay) was compared with the modelled number expected each week to determine if statistically significant excess mortality was seen in pilot and non-pilot areas [16]. This was applied to all-cause deaths and deaths where the primary cause of death was coded as respiratory applying the International Classification of Disease version 10 (ICD 10) code "J" [17]).

\section{Statistical methods}

For the RCGP, USISS sentinel and USISS mandatory schemes, cumulative disease incidence rates per 100,000 population by age and pilot group were calculated by summing the number of disease episodes each week from week 402014 to week 142015 relative to the average weekly population at risk, with exact Poisson confidence intervals (Cls) calculated.

For the RCGP swabbing and EDSSS schemes, cumulative influenza swab positivity and proportion of emergency department attendances coded as respiratory were calculated by age and pilot group by summing the number of positive samples/patients attending with respiratory symptoms and the number of samples tested each week/total number of attendances from week 402014 to week 142015 with exact binomial Cls calculated.

To determine the impact in primary and secondary school age pilot areas, odds ratios and corresponding $95 \% \mathrm{Cls}$ were calculated by age group and scheme, with non-pilot areas set as the reference group. Data were converted to binomial individual level across schemes and random effects logistic regression carried out, adjusting for clustering at the level of reporting unit (e.g. GP, trust, laboratory).

For the all-cause and respiratory coded deaths, the difference between the observed and expected weekly deaths was summed from week 402014 to week 142015 to obtain the cumulative number of excess deaths. Excess mortality rates were then calculated per 100,000 population.

\section{Laboratory methods}

Influenza laboratory confirmation for samples from primary and secondary care was undertaken using RT-PCR assays capable of detecting circulating influenza $A$ viruses, influenza $B$ viruses and other respiratory viruses. Samples in England were sent to the PHE 


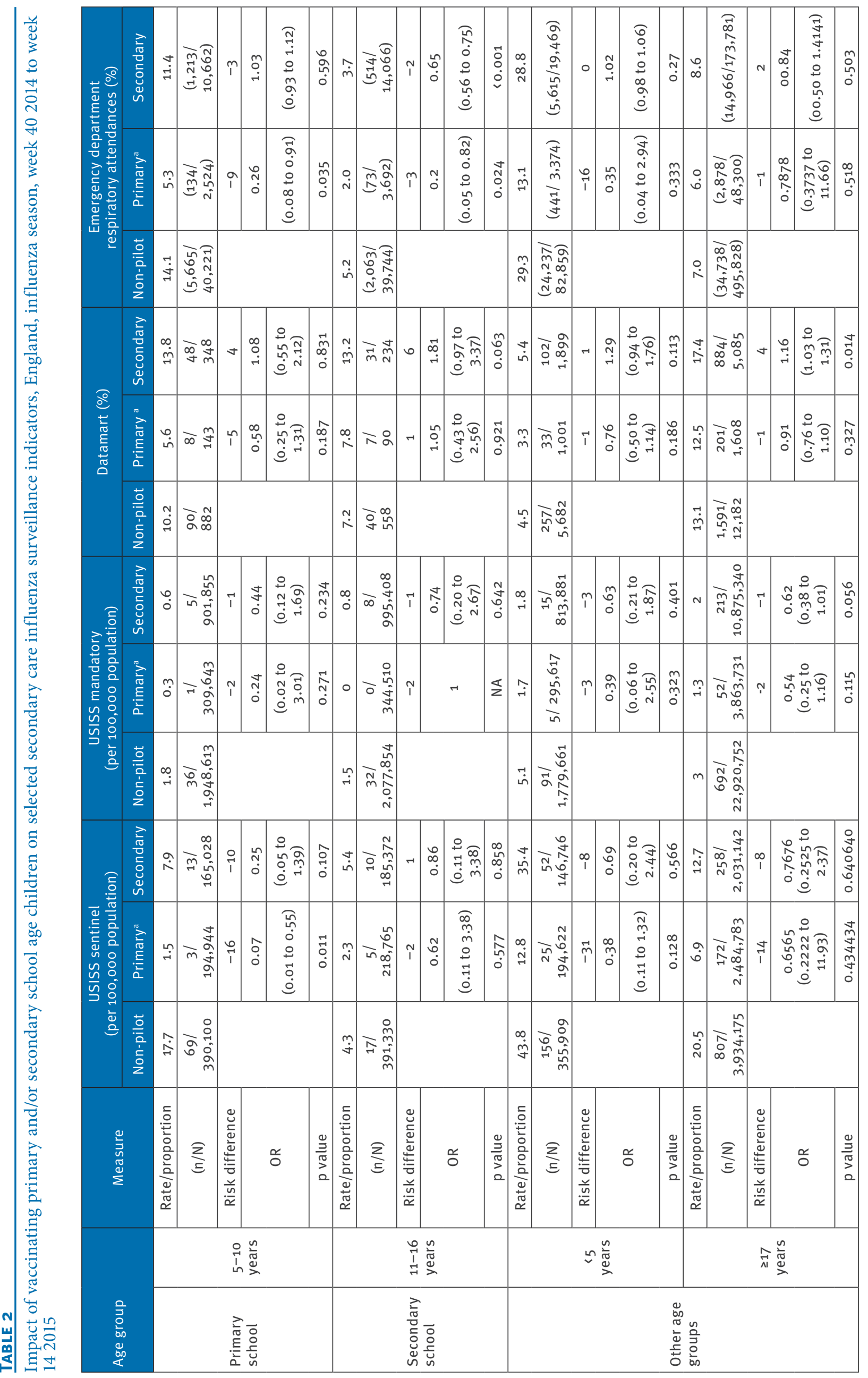


Microbiology Services, Colindale (RCGP scheme), one of the network of specialist PHE microbiology laboratories (SMN scheme) or NHS laboratories elsewhere in England.

\section{Results}

\section{Uptake}

The total target population for the pilots was estimated to be 346,962 primary school children and 371,109 for secondary school children aged 11 to13 years. Five of the six primary school pilot areas chose to deliver the programme through a school-based approach, while one, Cumbria, delivered through community pharmacies. Of the 12 secondary school pilots, 10 delivered the programme through schools only; one through community pharmacies and one through both schools and primary care.

An estimated 196,994 primary school age children received at least one dose of influenza vaccine resulting in an overall uptake of $56.8 \%$. This ranged from $32.3 \%$ to $63.1 \%$ at pilot-site level (Figure 2). An estimated 184,975 secondary school age children received at least one dose of influenza vaccine, an overall uptake of $49.8 \%$. Uptake ranged from $21.2 \%$ to $62.0 \%$ at pilot level (Figure 2).

In the primary school age programme, uptake in all the school-based areas was in excess of $50 \%$, compared with coverage of around $30 \%$ in the area delivering the programme through a pharmacy-based model. These findings were mirrored by the secondary school age programme, where uptake through school-based models was close to or well in excess of $50 \%$, with an uptake of less than $30 \%$ in the areas using communitybased delivery models (Figure 2).

Influenza vaccine uptake achieved through primary care in two to four year olds in primary school pilot areas only was $44.1 \%$ (224 practices) compared with $39.4 \%(2,361$ practices $)$ in secondary school age vaccine pilot areas; $35.1 \%$ (1,012 practices) in primary and secondary school pilot areas and $38.1 \%$ (4,176 practices) in non-pilot areas.

\section{Programme impact}

\section{Patterns of activity}

The cumulative ILI consultation rate and swab positivity in primary care, emergency department respiratory attendances, cumulative hospitalisation incidence rate, RDMS influenza positivity and ICU/HDU rates from week 402014 to week 142015 were generally lower in pilot areas where primary school age children were vaccinated compared with non-pilot areas across both targeted and non-targeted age groups (Figures 3 and 4). These differences were less marked for the cumulative ICU/HDU and RDMS indicators, particularly in the older non-targeted age group ( $\geq 17$ years of age).
A large excess all-cause and respiratory mortality was observed in both pilot and non-pilot areas. No significant reduction in all-cause mortality was observed in primary school pilot areas compared with non-pilot areas for all ages, whereas a significant reduction was observed for respiratory excess mortality (Figure 5).

Overall when comparing the various cumulative influenza indicators for secondary school pilot areas (11-13 year olds vaccinated only) to non-pilot areas, no such differences were observed, in both targeted and nontargeted age groups (Figures 3, 4 and 5).

Examination of pre-vaccination data for those indicators for which data were available, provided a mixed pattern, with evidence of similar activity in primary school areas compared to others in one season, whereas it was lower in another (Figure 6).

\section{Impact}

Vaccinating primary school age children resulted in significant reductions in cumulative incidence/laboratoryconfirmed positivity in the targeted age group (5-10 years) in pilot compared with non-pilot areas for GP ILI consultations ( $94 \%$ reduction, $p=0.018$ ); emergency department respiratory attendances ( $74 \%$ reduction, $\mathrm{p}=0.035$ ), confirmed influenza hospital admissions (93\% reduction, $p=0.012)$; with non-significant reductions in GP swabbing positivity (75\% reduction, $\mathrm{p}=0.213)$, confirmed influenza ICU admissions (76\% reduction, $p=0.271$ ) and DataMart influenza positivity ( $42 \%$ reduction. $\mathrm{p}=0.187$ ) (Tables 1,2 ).

Vaccinating primary school age children also resulted in an indirect non-significant reduction in under five year-olds, in pilot compared with non-pilot areas, for GP ILI consultations ( $92 \%$ reduction, $p=0.052$ ); emergency department respiratory attendances $(65 \%, p=0.33)$; confirmed influenza hospital admissions (61\% reduction, $p=0.128)$; confirmed influenza ICU/HDU admissions (61\% reduction, $p=0.324$ ) and DataMart influenza positivity $(24 \% . p=0.186)$ (Tables 1 and 2).

Significant indirect reductions were also seen in individuals $\geq 17$ years of age when comparing GP ILI consultations in primary school pilot to non-pilot areas (59\% reduction, $p=0.018$ ) and non-significant reductions in sentinel GP swabbing ( $32 \%$ reduction, $p=0.206)$; emergency department respiratory attendances (21\% reduction, $p=0.518)$; influenza confirmed hospital admissions ( $34 \%$ reduction, $p=0.434$ ); influenza confirmed ICU/HDU admissions ( $46 \%$ reduction, $p=0.115$ ) and DataMart influenza positivity ( $9 \%$ reduction; $\mathrm{p}=0.327$ )(Tables 1 and 2).

Vaccinating secondary school age-children aged 11-13 years of age alone did not result in a significant reduction in cumulative incidence/positivity for any surveillance indicator when comparing secondary school pilot areas to non-pilot areas, for both targeted and 
non-targeted age groups, with the exception of emergency department attendances in the target age group and confirmed influenza hospital admissions in adults (Table 2).

Through determining cumulative risk difference between pilot and non-pilot areas by age group and indicator, it is estimated that 16 primary school age children needed to be vaccinated to prevent one GP ILI consultation in the pilot population; 317 children to prevent one confirmed influenza hospitalisation and 2,205 children to prevent one confirmed influenza ICU/ HDU admission.

\section{Discussion}

This study assesses the uptake and evaluates the impact of the second season of the new UK LAIV programme for children in England. Piloting the LAIV programme in primary and now secondary school age children in the 2014/15 influenza season, resulted in similar or higher levels of uptake compared with the first. Pilot areas that chose to deliver the programme through school settings achieved higher uptake than those delivered through community settings, such as pharmacies. Despite the circulation of drifted $A\left(\mathrm{H}_{3} \mathrm{~N}_{2}\right)$ and $B$ influenza strains, our results demonstrate that vaccinating children of primary school age resulted in a significant reduction in incidence for a range of surveillance indicators. This effect was evident in targeted and non-targeted age groups compared with populations where primary school age children were not vaccinated. The size of the effect was less for more severe endpoints, in particular excess mortality. Vaccination of secondary school age children alone (11-13 years of age) failed to show conclusive evidence of such reductions in disease incidence in either targeted or nontargeted age-groups.

The study has a series of strengths; it builds on approaches developed in $2013 / 14$ to evaluate the uptake and impact of the newly established childhood LAIV programme, population-level data sources are used and the findings are consistent with the 2013/14 findings in terms of uptake, and an impact seen across a range of indicators when primary school children only were targeted [6]. There are, however, also some limitations. Firstly, examination of historical data suggests some caution is needed. The apparent effect sizes should not be overestimated. Our results suggest that the level of activity was lower for some indicators in the previous season $(2012 / 13)$ in primary school age pilot areas, although the observation was less apparent in the season before that (2011/12) (Figure 6). Secondly, uptake in two to four year-olds in primary school age only pilot areas was slightly different compared with non-pilot areas, which has the potential to affect effect sizes.

The uptake achieved in school age children this season builds on that reached in $2013 / 14$, with coverage now in excess of $50 \%$ in almost all pilot areas delivering the programme through a school-based approach (for either primary or secondary school age children). Earlier modelling work had suggested that at these levels of uptake for all school-age children, indirect benefits through reduction in population transmission of influenza are likely to occur [2]. Our findings also highlight the lower uptake among school age children achieved in areas deploying non-school based delivery approaches. This mirrors observations in relation to human papillomavirus (HPV) adolescent vaccine programmes, where countries using school-based delivery models achieved consistently higher uptake compared with other approaches [18]. In countries such as England, with its very high school attendance levels, there seem to be clear advantages to this delivery approach for a paediatric influenza vaccine programme for children of school age, although there was still variation in uptake, particularly in relation to factors such as deprivation and ethnicity in 2013/14 [19]. Further work is still required to refine the optimal delivery model, particularly from an equity and efficiency perspective.

The finding that vaccinating children of primary school age, in addition to the pre-school vaccination programme, led to reductions in disease incidence in both targeted and non-targeted age groups for a range of influenza indicators builds on observations from the first year of the programme when only primary school age children were vaccinated in pilot areas [6]. This season we observed in these areas decreases in influenza disease not only in the primary school age children themselves, but also indirectly in children under five years of age, where the burden of influenza is recognised to be highest, together with smaller indirect reductions in adults, where influenza disease burden is also high (in particular in the elderly and clinical risk groups). The indirect impact of vaccinating primary school age childrenunder five years old is over and above any direct impact that might have been due to the pre-school LAIV programme itself which operated across the whole of England in both pilot and non-pilot areas. These indirect reductions were consistently seen for primary care consultations (for both clinical and virological end-points) and laboratory confirmed hospitalisations and ICU/HDU admissions. As seen in 2013/14 though, the effect sizes became less as the end-points become more severe. These findings are supported by publications from elsewhere [3-5], including a recent article by Tran et al. showing that vaccination of school age children (with ca50\% uptake) led to large reductions in ILI emergency department visits across all ages in the local community [20]. In addition, the differential roll-out of the LAIV programme across the countries of the UK, with Scotland and Northern Ireland vaccinating primary school age children and Wales secondary school age children in 2014/15 has shown some early encouraging signs in relation to reductions in primary care consultations for those countries vaccinating primary school age children. This will provide further important opportunities to understand the population 
level impacts of the universal paediatric influenza vaccination programme [8].

The $2014 / 15$ season was characterised by significant excess all-cause mortality across Europe, particularly in the elderly, an observation consistent with the circulation of influenza $\mathrm{A}\left(\mathrm{H}_{3} \mathrm{~N}_{2}\right)$ [8]. Although there was reduction in respiratory excess mortality in primary compared with non-pilot areas, we found no evidence of a significant reduction in excess all-cause mortality. The reduction in excess respiratory mortality in those areas where primary school age children were vaccinated is encouraging, though the reasons for the lack of visibility of an indirect effect for excess all-cause mortality, which is a more non-specific indicator, are not totally clear, but could well be linked to lack of study power and is consistent with the smaller reductions we saw for the more severe end-points in the older agegroups. Further work is planned to understand these differences, as this is where much of the health economic benefits of a school-age influenza vaccination programme will be derived [2].

Despite looking at a range of indicators, we were unable to demonstrate evidence that vaccinating a cohort of children of secondary school age (albeit the first two years of secondary school) alone led to any consistent reduction in disease incidence in either targeted or non-targeted age groups in pilot areas. Although other studies have shown reductions in rates of respiratory illness in secondary school populations that undertake universal vaccination [21], no other studies, to our knowledge have shown population level benefits of vaccinating secondary school age children alone, although it is important to note that only two cohorts of secondary school age pupils were offered vaccination in the present pilot.

It is also important to note that the reductions we observed occurred in a season in which an antigenically and genetically drifted $\mathrm{A}\left(\mathrm{H}_{3} \mathrm{~N}_{2}\right)$ strain was the predominant circulating strain $[8,9]$, that had earlier resulted in low overall vaccine effectiveness against influenza $A\left(\mathrm{H}_{3} \mathrm{~N}_{2}\right)$, albeit with some evidence of effectiveness for LAIV in children [22]. Of further note, is that the dominant circulating influenza $B$ viruses at the end of the 2014/15 season also showed evidence of drift from the influenza B/Yamagata lineage vaccine strain [8]. These population-level impact findings of LAIV vaccination of school-age children suggests LAIV may have cross-protective effects against drifted strains, as reported previously [23].

In conclusion, our findings support the on-going roll out of the national LAIV programme for children of primary school age. The added benefit of vaccinating secondary school age children needs further careful consideration. Further work will need to continue to evaluate the impact of the LAIV programme against a range of end-points, in particular mortality related end-points.
Acknowledgements

The authors would like to acknowledge all the patients involved; the PHE and NHS staff in each of the vaccine pilot sites who were responsible for planning and delivering the LAIV -school age programme; all the participating general practices of the RCGP Research and Surveillance Centre Unit sentinel scheme; all participating emergency departments in the Emergency Department Syndromic Surveillance System and the Royal College of Emergency Medicine; all the participants of the UK Severe Influenza Surveillance Scheme; and the staff of the PHE Respiratory Virus Unit and contributing PHE and NHS laboratories. This work was supported by Public Health England as part an ongoing evaluation of the introduction of the new childhood influenza vaccination programme.

\section{Conflict of interest}

None declared.

Authors' contributions

RP led the design of the study and study group; all coauthors were members of the study group and involved in data collection, management and analyses; HG, NA and RP led the data analysis; HG undertook the summary analyses; $\mathrm{HZ}$ was responsible for the RDMS system, data management and analysis; NB was responsible for the USISS system, data management and analysis; HH, AE and GS were responsible for the EDSSS data system management and analysis; IY worked directly with the practices and SP, DM and SdeL were responsible for the RCGP data system management and analysis; JE, MD and $M Z$ were responsible for virological testing schemes; SS and NB were responsible for monitoring of vaccine uptake in the pilot sites; RP drafted the initial manuscript; all co-authors reviewed and commented including approval of the final version

\section{References}

1. Joint Committee on Vaccination and Immunisation. Meeting minute.5 Oct 2011. London. Available from: http://webarchive. nationalarchives.gov.uk/20120907090205/http://www.dh.gov. uk/prod_consum_dh/groups/dh_digitalassets/@dh/@ab/ documents/digitalasset/dh_133598.pdf

2. BaguelinM, FlascheS, CamachoA, DemirisN, MillerE, EdmundsWJ. Assessing optimal target populations for influenza vaccination programmes: an evidence synthesis and modelling study.PLoS Med. 2013;10(10):e1001527. DOI: 10.1371/journal.pmed.1001527 PMID: 24115913

3. GrijalvaCG, ZhuY, SimonsenL, MitchelE, GriffinMR. The population impact of a large school-based influenza vaccination campaign.PLoS ONE. 2010;5(11):e15097. DOI: 10.1371/journal.pone.0015097 PMID: 21209872

4. KingJC, CummingsGE, Stoddard,, ReadmondBX, MagderLS, StongM, et al. A pilot study of the effectiveness of a school-based influenza vaccination program. Pediatrics. 2005;116(6):e868-73. DOI: 10.1542/peds.2005-1301 PMID: 16322144

5. KingJC, LichensteinR, CummingsGE, MagderLS. Impact of influenza vaccination of schoolchildren on medical outcomes among all residents of Maryland.Vaccine. 2010;28(49):7737-42. DOI: 10.1016/j.vaccine.2010.09.064 PMID: 20933566

6. PebodyRG, GreenHK, AndrewsN, ZhaoH, BoddingtonN, BawaZ, et al. Uptake and impact of a new live attenuated influenza vaccine programme in England: early results of a pilot in primary school-age children, 2013/14 influenza season. Euro Surveill. 2014;19(22):20823. DOI: 10.2807/1560-7917. ES2014.19.22.20823 PMID: 24925457

7. Department of Health. Public Health England (PHE). The national flu immunisation programme 2014/15. London: PHE. $28 \mathrm{Apr} 2014$. Available from: https://www.gov.uk/government/ uploads/system/uploads/attachment_data/file/316007/ FlulmmunisationLetter2014_accessible.pdf 
8. Public Health England (PHE). Surveillance of influenza and other respiratory viruses in the United Kingdom: winter 2014 to 2015. London: PHE. May 2015. Available from: https://www. gov.uk/government/uploads/system/uploads/attachment_ data/file/429617/Annualreport_March2015_ver4.pdf

9. WHO European Region and the European Influenza Surveillance Network,BrobergE, SnackenR, AdlhochC, BeautéJ, GalinskaM, PereyaslovD, et al. . Start of the 2014/15 influenza season in Europe: drifted influenza $\mathrm{A}\left(\mathrm{H}_{3} \mathrm{~N}_{2}\right)$ viruses circulate as dominant subtype.Euro Surveill. 2015;20(4):21023. DOI: 10.2807/1560-7917.ES2015.20.4.21023 PMID: 25655052

10. BolotinS, PebodyR, WhitePJ, McMenaminJ, PereraL, NguyenVan-TamJS, et al. A new sentinel surveillance system for severe influenza in England shows a shift in age distribution of hospitalised cases in the post-pandemic period. PLOS ONE. 2012;7(1):e30279. DOI: 10.1371/journal.pone.0030279 PMID: 22291929

11. Public Health England (PHE). Eastern Region Public Health Observatory (Erpho). Acute hospital catchment populations 2009. London: PHE. [Accessed 16 Jun 2015]. Available from: http://www.erpho.org.uk/viewResource.aspx?id=21919

12. Office for National Statistics. Population Estimates for UK, England and Wales, Scotland and Northern Ireland, Mid-2013 - SUPERSEDED. London: Office for National Statistics. 26 Jun 2014. Available from: http:// www.ons.gov.uk/ons/publications/re-reference-tables. html?edition $=$ tcm $\% 3 A 77-322718$

13. ZhaoH, GreenH, LackenbyA, DonatiM, Ellis], ThompsonC, et al. A new laboratory-based surveillance system (Respiratory DataMart System) for influenza and other respiratory viruses in England: results and experience from 2009 to 2012. Euro Surveill. 2014;19(3):20680. DOI: 10.2807/1560-7917. ES2014.19.3.20680 PMID: 24480060

14. ElliotAJ, HughesHE, HughesTC, LockerTE, ShannonT, HeyworthJ, et al. Establishing an emergency department syndromic surveillance system to support the London 2012 Olympic and Paralympic Games. Emerg Med J. 2012;29(12):954-60. DOI: 10.1136/emermed-2011-200684 PMID: 22366039

15. NielsenJ, MazickA, AndrewsN, DetsisM, FenechTM, FloresVM, et al. Pooling European all-cause mortality: methodology and findings for the seasons 2008/2009 to 2010/2011. Epidemiol Infect. 2013;141(9):1996-2010. DOI: 10.1017/ So950268812002580 PMID: 23182146

16. MazickA, GergonneB, NielsenJ, WuillaumeF, VirtanenMJ, FouilletA, et al. Excess mortality among the elderly in 12 European countries, February and March 2012. Euro Surveill. 2012;17(14):20138.PMID: 22516003

17. World Health Organization (WHO). International statistical classification of diseases and related health problems. - 10th revision. Geneva: WHO; 2010. Available from: www.who.int/ classifications/icd/ICD10Volume2_en_2010.pdf

18. ReganDG, HockingJS. Greatest effect of HPV vaccination from school-based programmes.Lancet Infect Dis. 2015;15(5):497-8. DOI: 10.1016/S1473-3099(15)70078-2 PMID: 25744473

19. GreenHK, AndrewsN, LetleyL, SunderlandA, WhiteJ, PebodyR. Phased introduction of a universal childhood influenza vaccination programme in England: population-level factors predicting variation in national uptake during the first year, 2013/14.Vaccine. 2015;33(22):2620-8. DOI: 10.1016/j. vaccine.2015.03.049 PMID: 25835576

20. TranCH, SugimotoJD, PulliamJR, RyanKA, MyersPD, CastlemanJB, et al. School-located influenza vaccination reduces community risk for influenza and influenza-like illness emergency care visits. PLoS ONE. 2014;9(12):e114479. DOI: 10.1371/journal.pone.0114479 PMID: 25489850

21. BrousseauN, GreenHK, AndrewsN, PryseR, BaguelinM, SunderlandA, et al. Impact of influenza vaccination on respiratory illness rates in children attending private boarding schools in England, 2013-2014: a cohort study. Epidemiol Infect. 2015;Apr 16:1-11. DOI: 10.1017/So950268815000667 PMID: 25876454

22. PebodyRG, WarburtonF, AndrewsN, Ellis), von WissmannB, RobertsonC, et al. Effectiveness of seasonal influenza vaccine in preventing laboratory-confirmed influenza in primary care in the United Kingdom: 2014/15 end of season results. Euro Surveill. 2015;20(36):21237.

23. NicholKL, MendelmanPM, MallonKP, JacksonLA, GorseGJ, BelsheRB, et al. Effectiveness of live, attenuated intranasal influenza virus vaccine in healthy, working adults: a randomized controlled trial. JAMA. 1999;282(2):137-44.DOI: 10.1001/jama.282.2.137 PMID: 10411194 